\title{
Theoretical Analysis of Strong-Axis Bending Mode Vibrations for Resonant Microcantilever (Bio)Chemical Sensors in Gas or Liquid Phase
}

\author{
Isabelle Dufour, Stephen M. Heinrich, and Fabien Josse, Senior Member, IEEE
}

\begin{abstract}
The frequency stability, sensitivity, and limit of detection of a coated-cantilever chemical sensor operating in a dynamic mode are mainly determined by its mechanical quality factor. While a coated-cantilever operating in the gas phase exhibits a large reduction in quality factor, immersion in liquids results in an even greater reduction in the $Q$-factor due to displaced fluid mass and losses in the surrounding liquid. In this paper, two different bending vibration modes are studied in order to minimize both the losses induced by the surrounding medium and the displaced fluid mass, thus increasing the quality factor and sensitivity and improving (decreasing) the detection limit of the biochemical microsensor. The two particular vibration modes both involve "first mode" flexural vibrations (but in different orthogonal planes), and are referred to herein as "weak-axis bending" (WAB) and "strong-axis bending" (SAB). Using Sader's model, the expressions for both the quality factor and the resonant frequency are analyzed for the case of immersion in a viscous fluid. The results indicate that the strong-axis bending mode has certain advantages over the more conventional weak-axis mode in enhancing the sensor sensitivity and detection limit, even for the case in which the WAB and SAB devices have identical resonant frequencies.

[1464]
\end{abstract}

Index Terms-Liquid environment, microcantilever sensors, quality factor effect, resonant frequency.

\section{INTRODUCTION}

$\mathbf{T}$ HE use of silicon microcantilevers as transducers in physical and biochemical sensing systems has increased in recent years [1]-[11]. In fact, the large ratio of surface area to mass makes the microcantilever extremely sensitive to surface processes. For biochemical detection, the microcantilever is covered with a biochemically sensitive coating that aims to selectively sorb the analyte or molecule of interest. The sorbed molecules can then be detected by monitoring the mechanical resonant frequency of the microcantilever. This frequency depends on the mass loading due to molecule adsorption by the sensitive coating(s). By monitoring the change in resonant frequency, one may deduce the mass of sorbed target molecules in

Manuscript received November 15, 2004; revised August 3, 2006. Subject Editor T. Kenny.

I. Dufour is with the IXL Laboratory, CNRS UMR5818, ENSEIRB/Université Bordeaux 1, 33405 Talence Cedex, France (e-mail: dufour@ixl.fr).

S. M. Heinrich is with the Department of Civil and Environmental Engineering, Marquette University, Milwaukee, WI 53201-1881 USA (e-mail: stephen.heinrich@marquette.edu).

F. Josse is with the Microsensor Research Laboratory and Department of Electrical and Computer Engineering, Marquette University, Milwaukee, WI 53201-1881 USA (e-mail: fabien.josse@marquette.edu).

Digital Object Identifier 10.1109/JMEMS.2006.885850 the sensitive coating and the concentration of target molecules in the surrounding medium. As with all frequency-output sensors, the limit of detection (LOD) of microcantilever sensors is mainly determined by the mechanical quality factor $Q$; a large $Q$ value means good frequency stability due to a minimization of the noise associated with the microcantilever and the oscillation driving circuit.

Since chemical sensors operate in either a gas or liquid medium, the $Q$ value of the coated-resonant microstructure is less than that of classical microelectromechanical systems (MEMS) operating in vacuum [12], [13]. In gas, the resonant frequencies may be reduced by a few percent, whereas $Q$ may exhibit reductions of two orders of magnitude from the value in vacuum [14]. Immersion in liquid results in even greater changes to the frequency response, with resonant frequencies and $Q$ values being an order of magnitude smaller than their values in the gas phase. The reduced value of $Q$, due to losses in the surrounding medium and displaced fluid mass (especially in liquids), directly affects the sensitivity and detection limit of these types of sensors.

The aim of this paper is to study two different bending vibration modes to understand their influence on the sensor sensitivity and to reduce both the losses induced by the surrounding medium and the displaced fluid mass, thus increasing the quality factor and sensitivity, while reducing the LOD. The two particular vibration modes considered both involve "first mode" flexural vibrations, but about two different axes of the beam cross-section. The first, and most often used, mode will be denoted as "weak-axis bending" (WAB). This mode involves bending about the axis on the beam's cross-section for which the flexural stiffness is a minimum [Fig. 1(a)]. For "strong-axis bending" ( $\mathrm{SAB}$ ), the bending occurs about the section's axis for which the flexural stiffness is a maximum [Fig. 1(b)]. While the WAB mode may be more easily excited than the SAB mode, the latter's more "streamlined" orientation in a fluid is expected to involve smaller losses and less movement of the surrounding fluid mass; thus, SAB may have some advantages over the WAB mode. Here we wish to examine these potential advantages in detail.

In the first part of this paper (Section II), previous theoretical results are summarized for beam vibrations in viscous fluids. These results include formulas for resonant frequency, forces associated with the surrounding fluid, quality factor, and frequency shift caused by the added mass of the sorbed target molecules. In Section III, results are presented for quantifying the sensitivity and detection limit of microcantilever sensors, as 
(a)

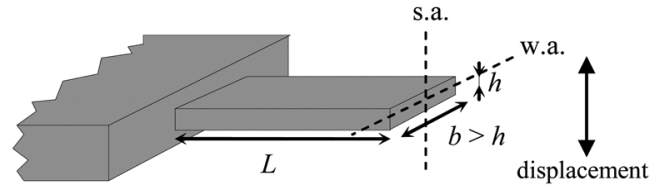

(b)

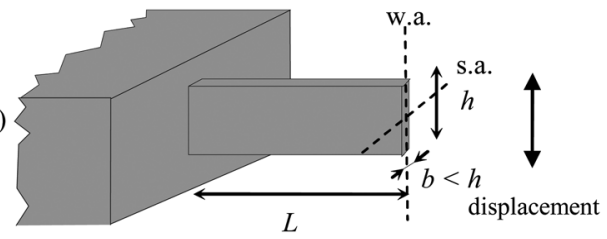

Fig. 1. (a) Weak-axis bending (WAB) and (b) strong-axis bending ( $\mathrm{SAB}$ (w.a. and s.a. are, respectively, the weak and strong axes of the cross-section).

these practical issues are directly related to the vibrational behavior of the microstructures. A comparison of sensor performance in both WAB and SAB modes is made in Section IV. In particular, the effects of the different modes on the resonant frequency, quality factor, sensitivity, and LOD are examined. The results indicate that the strong-axis bending mode has certain advantages over the more conventional weak-axis mode in enhancing the sensor sensitivity and detection limit.

\section{BEAM MECHANICS}

\section{A. Modeling Assumptions}

The theoretical results summarized in this paper are based on the following assumptions.

1) Only first-mode flexural vibrations are considered, regardless of the direction of bending (WAB or SAB).

2) The assumptions of classical beam theory are assumed to be valid [15].

3) Energy losses associated with the beam, including its sensitive coating and the support, are neglected in comparison with losses in the surrounding fluid.

4) The sensitive coating is assumed to have a negligible effect on a) the beam's bending stiffness and b) the fluid forces on the beam.

5) The beam's frequency shift is due to the sorbed target molecules in the coating, and the effects of the molecules on the beam's damping and stiffness properties are neglected.

\section{B. Resonant Frequency in Viscous Fluid}

Consider the beam parameters $L, h$, and $b$ defined in Fig. 1 . We shall assume that the beam vibrates in the vertical plane; however, the SAB mode of Fig. 1(b) corresponds to the lateral (horizontal) bending mode in Fig. 1(a). When a microcantilever beam vibrates in a viscous fluid, the fluid offers resistance to the motion. The fluid loading on the beam includes an inertial force, proportional to the beam acceleration, and a viscous or dissipative force that is proportional to the cantilever velocity [12], [16], [17]. These fluid effects will influence the dynamic response of the beam, in particular, the resonant frequency $f_{r}$ of the microcantilever immersed in a viscous fluid [17]

$$
f_{r}=f_{0} \frac{1}{\sqrt{1+\frac{L g_{2}}{M}}} \sqrt{1-\frac{1}{2 Q^{2}}} .
$$

Here $f_{0}$ is the undamped natural frequency of the microcantilever in vacuum, $Q$ is the quality factor of the cantilever/fluid system (depending on the losses), $g_{2}$ is a fluid-dependent inertia parameter, and $M$ is the mass of the cantilever. (Detailed expres- sions for $Q$ and $g_{2}$ will be provided shortly.). Should the microcantilever include one or more coatings, then mass $M$ should be interpreted as $M=m+m_{c}$, where $m$ and $m_{c}$ are the masses of the beam substrate and coating(s), respectively. The classical result for the natural frequency $f_{0}$ in vacuum is, e.g., [18]

$$
f_{0}=\frac{\lambda_{0}^{2}}{2 \pi L^{2}} \sqrt{\frac{\hat{E} I L}{M}}
$$

with $\lambda_{0}=1.875$ corresponding to the fundamental flexural mode and $I$ representing the cross-section's area moment of inertia taken about the horizontal axis of bending, given by $I=$ $b h^{3} / 12$. Parameter $I$ may be interpreted as the cross-section's resistance to bending about a particular axis. Symbol $\hat{E}$ represents the effective Young modulus, defined via curve-fitting of finite-element results as [19]

$$
\hat{E}=E\left[1+\left(1-e^{-1.5(b / L)}\right)\left(\frac{1}{1-\nu^{2}}-1\right)\right] .
$$

\section{Fluid Losses and Quality Factor}

The quality factor $Q$ of a microcantilever sensor in a fluid environment depends on the energy losses of the system. In general, these losses may be intrinsic (thermoelastic losses, clamping losses, bulk internal friction other than thermoelastic dissipation, etc.) [13] or due to the surrounding medium (viscous losses). In the case of (bio)chemical sensors, the surrounding medium is usually either a gas at atmospheric pressure or a liquid. Consequently, the losses due to viscous damping in the fluid will be dominant [12], in which case the results of Sader [17] will be applicable. Using the current notation, Sader's expression for $Q$ may be written as

$$
Q=\frac{2 \pi \sqrt{1+\frac{L g_{2}}{M}}}{\frac{L g_{1}}{M}} f_{0}
$$

where $g_{1}$ and $g_{2}$ are the fluid-dependent viscosity and inertia parameters, given explicitly by

$$
g_{1}=\pi \eta R_{e} \Gamma_{i}\left(R_{e}\right), \quad g_{2}=\frac{\eta R_{e}}{2 f_{r}} \Gamma_{r}\left(R_{e}\right) .
$$

Parameter $\eta$ is the fluid viscosity, $R_{e}$ is the Reynolds number for the flow, and $\Gamma_{r}$ and $\Gamma_{i}$ are the real and imaginary parts of the (dimensionless) "hydrodynamic function"

$$
\Gamma\left(R_{e}\right)=\Omega\left(R_{e}\right)\left[1+\frac{4 i K_{1}\left(-i \sqrt{i R_{e}}\right)}{\sqrt{i R_{e}} K_{0}\left(-i \sqrt{i R_{e}}\right)}\right] .
$$


The Reynold's number is defined as

$$
R_{e} \equiv \frac{\pi \rho_{f} b^{2} f_{r}}{2 \eta}
$$

where $\rho_{f}$ is the fluid's mass density. In (4), the term $L g_{1} / M$ is due to the dissipative action of the fluid (viscous losses) and the term $L g_{2} / M$ is associated with the additional mass due to the displacement of surrounding fluid. In (6), $K_{0}$ and $K_{1}$ are modified Bessel functions and $\Omega\left(R_{e}\right)$ is a correction function associated with the rectangular beam cross-section [17]. According to Sader, the range of applicability for this representation is $R_{e} \in\left[10^{-6}, 10^{4}\right]$, which is based on matching numerical results for flow across an infinitely thin rectangular beam.

\section{Frequency Shift}

When a coated resonant microcantilever is placed in a gas or liquid environment, some of the target molecules are sorbed into the sensitive layer. As a result, the microcantilever's mass, stiffness, losses, and the additional equivalent mass due to fluid may be modified. The associated frequency shift $\Delta f_{r}$ can be expressed in terms of the mass variation, stiffness variation, etc. Assuming that these variations are small, (1) and (2) yield the following expression for the relative frequency shift:

$$
\begin{aligned}
\frac{\Delta f_{r}}{f_{r}} \approx \frac{\Delta k}{2 k} & +\frac{\Delta Q}{Q\left(2 Q^{2}-1\right)} \\
& -\frac{L \Delta g_{2}}{2\left(M+L g_{2}\right)}+\frac{\Delta M}{2 M}\left(-1+\frac{L g_{2}}{M+L g_{2}}\right)
\end{aligned}
$$

where $k \equiv 3 \hat{E} I / L^{3}$ has been introduced to denote the stiffness of a cantilever beam under an end force. The variations of the stiffness, quality factor, and inertial drag force caused by the sorbed molecules are generally negligible so that the frequency shift is essentially due to the mass variation $\Delta M$. Using (1), the frequency shift becomes

$$
\begin{aligned}
\Delta f_{r} & =-\frac{\Delta M}{2 M\left(1+\frac{L g_{2}}{M}\right)} f_{r} \\
& =-\frac{\Delta M}{2 M\left(1+\frac{L g_{2}}{M}\right)^{3 / 2}} f_{0} \sqrt{1-\frac{1}{2 Q^{2}}} .
\end{aligned}
$$

In a gas phase, the term due to the inertial part of the drag force $L g_{2} / M$ can be neglected because the "equivalent mass" due to the fluid $\left(L g_{2}\right)$ is usually very small compared to the cantilever mass $M$. But as will be seen in Section IV, in a liquid medium this term should be included as it is not always negligible.

\section{Practical Issues For MicrocantileVer Sensors}

\section{A. Sensor Sensitivity}

In terms of the (dimensionless) partition coefficient $K$ of the coating/analyte pair in a given fluid environment, the variation of the sensor's mass is given by [20]

$$
\Delta M=K h_{c} A_{c} C_{A}
$$

in which $h_{c}$ is the sensitive coating thickness, $A_{c}$ the coated surface area of the microcantilever, $C_{A}$ the analyte concentration in the fluid (mass per unit volume), and the partition coefficient $K$ is a measure of the distribution of the analyte between the sorbed phase and the surrounding medium. Then, under the assumption $m_{c} / m<<1$, the sensor sensitivity (frequency shift per unit analyte concentration) due to analyte sorption reduces to the following linear relationship between sensitivity and the ratio of coating volume $\left(V_{c}=h_{c} A_{c}\right)$ to the microstructure volume $(V=b h L)$ :

$$
S \equiv\left|\frac{\Delta f_{r}}{C_{A}}\right|=\frac{K f_{r}}{2 \rho\left(1+\frac{L g_{2}}{m}\right)} \frac{V_{c}}{V} .
$$

Here $\rho$ is the mass density of the beam's substrate material. Equation (11) shows that sensitivity may be enhanced by increasing resonant frequency (by increasing the bending stiffness or the quality factor) or by decreasing the equivalent fluid mass. All of these benefits may be achieved by employing the SAB mode. (See Section IV.)

\section{B. Detection Limit of Sensor}

In an oscillator configuration, the frequency shift due to the phase noise $\Delta \theta_{n}$ of the driving electronic circuit may be written as [21]

$$
\Delta f_{\text {noise }} \approx \frac{f_{r} \Delta \theta_{n}}{2 Q} .
$$

Note that $\Delta f_{\text {noise }}$ is proportional to the resonant frequency and inversely related to $Q$. Thus, higher resonant-frequency microcantilevers will have inherently higher LOD values unless accompanied by higher $Q$ values. (Later, we shall see that the higher $Q$ value in SAB microcantilevers more than compensates for the higher resonant frequency, especially in liquid environments). Equation (12) may be used to determine the signal-tonoise ratio

$$
\left|\frac{\Delta f_{r}}{\Delta f_{\text {noise }}}\right| \approx \frac{\Delta M}{M\left(1+\frac{L g_{2}}{M}\right)} \frac{Q}{\Delta \theta_{n}} .
$$

The limit of detection of the sensor $C_{A \text { min }}$ is usually defined as the analyte concentration corresponding to a frequency shift equal to three times the frequency noise of the system measurement [22]. Thus, under the assumption that $m_{c} / m<<1$, (10) and (13) may be combined to yield the sensor's LOD:

$$
C_{A \min }=\frac{3 \rho\left(1+\frac{L g_{2}}{m}\right) \Delta \theta_{n}}{K Q\left(\frac{V_{c}}{V}\right)} .
$$

As was the case with the sensitivity, the LOD may be improved by using SAB in place of the WAB mode to increase the quality factor or decrease the equivalent mass due to fluid. In addition, (14) clearly shows that the LOD is inversely related to the volume fraction of the coating material, regardless of the mode of operation. 
TABLE I

MicrocantileVER GEOMETRIES FOR NUMERICAL SimULATIONS ( $g=$ GAS MEdium, $l=$ LiQuid Medium)

\begin{tabular}{|c|c|c|c|c|c|}
\hline Case & Mode & Medium & $\begin{array}{c}b \\
(\mu \mathrm{m})\end{array}$ & $\begin{array}{c}h \\
(\mu \mathrm{m})\end{array}$ & $\begin{array}{c}L \\
(\mu \mathrm{m})\end{array}$ \\
\hline $1 g$ & WAB & air & 50 & 3 & 400 \\
\hline $2 g$ & SAB & air & 3 & 50 & 400 \\
\hline $3 g$ & WAB & air & 50 & 3 & 98.8 \\
\hline $1 l$ & WAB & water & 50 & 3 & 400 \\
\hline $2 l$ & SAB & water & 3 & 50 & 400 \\
\hline $3 l$ & WAB & water & 50 & 3 & 61.5 \\
\hline
\end{tabular}

\section{COMPARISON OF WAB AND SAB SIMULATIONS}

General results for the vibration of a microcantilever sensor in a fluid have been summarized and discussed in the preceding sections. Next we shall apply these general results to make a numerical comparison between the performance parameters in $\mathrm{WAB}$ and $\mathrm{SAB}$ modes of operation. In particular, we wish to focus the discussion on the values of resonant frequency, quality factor, sensitivity, and limit of detection.

Numerical results will be presented for WAB and SAB operation in both gas and liquid environments. The media used in the simulations are air $\left(\rho_{f}=1.29 \mathrm{~kg} / \mathrm{m}^{3}, \eta=1.8 \times 10^{-5} \mathrm{~kg} / \mathrm{m}-\mathrm{s}\right)$ and water $\left(\rho_{f}=997 \mathrm{~kg} / \mathrm{m}^{3}, \eta=8.610^{-4} \mathrm{~kg} / \mathrm{m}\right.$-s $)$. Silicon is taken as the substrate material $\left(\rho=2330 \mathrm{~kg} / \mathrm{m}^{3}, E=150 \mathrm{GPa}\right.$, $\nu=0.273)$, while the coating material $\left(\rho_{c}\right)$ and coating geometry $\left(h_{c}, A_{c}, V_{c}\right)$ are arbitrary provided that $m_{c} / m \ll 1$.

In all of the simulations, the cross-sectional dimensions of the microcantilever are fixed. Therefore, the bending mode (WAB or $\mathrm{SAB}$ ) is completely determined by the orientation of this cross-section relative to the direction of bending. Assuming that all vibrations occur in the vertical direction (see Fig. 1), cases for which $b>h(b<h)$ will therefore result in weak (strong)-axis bending. Definitions of the various cases used in the simulations are included in Table I. Symbols " $g$ " and " $l$ " in the case numbering system denote "gas" and "liquid" environments, respectively. In each medium, the beam lengths in cases 1 and 2 are identical, while in cases $3 l$ and $3 g$ (both WAB), the beam lengths are specified in such a way that these systems will have the same resonant frequencies as their case 2 (SAB) counterparts. This will enable us to compare nonfrequency effects in the $\mathrm{WAB}$ and $\mathrm{SAB}$ modes.

Based on the results of the simulations (see Table II), the following observations may be made.

- Cases $1 g-3 g$ illustrate the well-known result that the resonant frequency $f_{r}$ in air is only slightly smaller than the natural frequency in a vacuum $\left(f_{0}\right)$. However, when the microcantilever operates in the WAB mode in water (cases $1 l$ and $3 l$ ), we see a dramatic drop (more than $60 \%$ ) in the resonant frequency compared with the natural frequency in vacuum. This drop is due to the viscous losses and effective mass associated with the surrounding fluid. When the microcantilever vibrates in the SAB mode (case $2 l$ ), the resonant frequency undergoes only a $2 \%$ drop from $f_{0}$ because of the more "streamlined" orientation.

- For fixed beam dimensions $(b, h, L)$, a SAB mode of operation results in significant enhancements in $Q$, sensitivity,
TABLE II

RESULTS OF NUMERICAL SIMULATIONS

\begin{tabular}{|c|c|c|c|c|c|c|}
\hline Case & Mode & $\begin{array}{c}f_{0} \\
(\mathrm{kHz})\end{array}$ & $\begin{array}{c}f_{r} \\
(\mathrm{kHz})\end{array}$ & $Q$ & $\begin{array}{c}\frac{S}{K\left(\frac{V_{c}}{V}\right)} \\
\left(\frac{\mathrm{Hz}}{\mathrm{kg} / \mathrm{m}^{3}}\right)\end{array}$ & $\begin{array}{c}\frac{C_{A \min }}{\frac{\Delta \theta_{n}}{K}\left(\frac{V}{V_{c}}\right)} \\
\left(\mathrm{kg} / \mathrm{m}^{3}\right)\end{array}$ \\
\hline $1 g$ & WAB & 24.5 & 24.3 & 115 & 5.1 & 62 \\
\hline $2 g$ & SAB & 405 & 405 & 5050 & 86.9 & 1.4 \\
\hline $3 g$ & WAB & 407 & 405 & 501 & 86.1 & 14 \\
\hline $1 l$ & WAB & 24.5 & 7.77 & 3 & 0.2 & 19100 \\
\hline $2 l$ & SAB & 405 & 397 & 41 & 81.6 & 177 \\
\hline $3 l$ & WAB & 1057 & 396 & 16 & 12.0 & 3010 \\
\hline
\end{tabular}

and LOD as compared to the WAB mode. This is true for both the gas and liquid. (See cases $1 g, 2 g$, and $1 l, 2 l$, respectively.)

- Comparisons between cases $2 g$ and $3 g$ and between cases $2 l$ and $3 l$ show that improvements due to the SAB mode are associated with more than just the increase in resonance frequency. (Recall that WAB cases $3 g$ and $3 l$ were chosen to have the same resonant frequencies as their $\mathrm{SAB}$ counterparts.) These non-frequency-related advantages of $\mathrm{SAB}$ over WAB will be discussed in more detail in the following bullet items.

- An examination of cases $2 g$ and $3 g$ shows that the SAB mode yields a tenfold improvement in quality factor and LOD over the WAB mode in air, while the sensitivity is essentially unchanged. This latter result is to be expected because the sensitivity, as defined in (11), depends on resonant frequency and the fluid mass term $\mathrm{Lg}_{2} / \mathrm{m}$; the resonant frequency is the same for these two cases, while $L g_{2} / m$ is negligible in both cases (i.e., small relative to unity) because of the gas environment. (See Table III.) The tenfold improvements in $Q$ and LOD (case $2 g$ versus $3 g$ ) are directly related to the tenfold reduction in the viscous loss term $L g_{1} / m$ when the mode is changed from WAB to SAB. (See Table III and (4) and (14).)

- A comparison of cases $2 l$ and $3 l$, which have the same resonant frequency $f_{r}$, shows that in water the SAB mode yields an improvement in $Q$ by a factor of 2.6 and in LOD by a factor of 17 . Moreover, despite the identical resonant frequencies, the SAB mode is about seven times more sensitive than the WAB mode in water. Unlike in gases, the sensitivity in liquids is not driven solely by the resonant frequency. It also depends on the value of $L g_{2} / m$ (fluid mass term), which may be significant in liquids, especially in the WAB mode. (See Table III and (11).) Intuitively, we expect that the SAB mode will move less fluid mass than the WAB mode because of its more "streamlined" orientation. The results presented here help to quantify this advantage.

- For the illustrative examples considered, the SAB mode results in negligible values of the equivalent fluid mass term in both gas and liquid (0.0001 and 0.04, respectively). This term is very significant for the WAB mode in a liquid (values of 8 and 6 in Table III) and should not be neglected in calculating the values of frequency, sensitivity, and limit of detection. 
TABLE III

FLUID EFFECTS

\begin{tabular}{|c|c|c|c|c|c|c|}
\hline Case & $1 g$ & $2 g$ & $3 g$ & $1 l$ & $2 l$ & $3 l$ \\
\hline Mode & WAB & SAB & WAB & WAB & SAB & WAB \\
\hline$L g_{1} / m(\mathrm{kHz})$ & 1.3 & 0.5 & 5.1 & 136 & 63 & 1073 \\
\hline$L g_{2} / m$ & 0.015 & 0.0001 & 0.010 & 8 & 0.04 & 6 \\
\hline
\end{tabular}

- Comparisons between cases $1 g$ and $1 l$ and between cases $2 g$ and $2 l$ lead to the conclusion that the frequency drop for $\mathrm{SAB}$ going from gas to liquid is relatively small compared to the frequency drop in the WAB mode (Table II). In fact, although $Q$ and $L g_{2} / m$ (Table III) appear to get much worse in going from $2 g$ to $2 l$, their contributions are still relatively small in the frequency equation [(1)], i.e., $1 /\left(2 Q^{2}\right)$ and $L g_{2} / m$ are still much less than one. The fact that these two terms are negligible in the SAB mode, even in liquid, is the reason why the frequency drop is small in going from $2 g$ to $2 l$. The same remark is true regarding the sensitivity, i.e., the large drop in $Q$ only results in a small decrease in normalized sensitivity. However, there is a larger increase in the LOD for SAB (compared with sensitivity) in going from air to water because LOD is inversely related to $Q$. Nevertheless, this increase in LOD is still much smaller than if the WAB mode were used.

In order to have a better understanding of the sensor performance in the SAB mode, some numerical results for the nonnormalized sensitivity and LOD are given in Table IV. These are directly deduced from the normalized values of Table II combined with specified properties of the coated microstructure. In particular, the results of Table IV are based on a sensitive coating having a $1-\mu \mathrm{m}$ thickness placed on one of the two wider surfaces of the microcantilever. Using the same geometric parameters as specified in Table I, this results in a coating-to-microstructure volume ratio of $V_{c} / V=1 / 3$. The sensitive coating for the detection of toluene is polydimethylsiloxan (PDMS), having partition coefficients of 1164 and 279 in air and water, respectively [20], [23], [24]. The variation of the amplifier phase noise has been taken to be $\Delta \theta_{n}=0.001 \mathrm{rad}$. While these results allow us to make a comparison between the SAB and WAB modes, we recognize that the individual performances of these microsensors may be improved by modifying the oscillator phase stability and by increasing the sensitive coating volume in appropriate ways (e.g., by coating all free surfaces of the microcantilever). Here we also emphasize that the unit of " $\mathrm{kg} / \mathrm{m}^{3}$ " in Table II has been converted to "ppm" in Table IV, as this is a more commonly used unit in practical sensing applications. Therefore, one should not compare sensitivity and LOD values between liquid and gas in Table IV because the ppm values are with respect to different media.

The results of Table IV indicate the following.

- Switching the orientation of a given microbeam from WAB to $\mathrm{SAB}$ results in significant improvements in sensitivity and LOD. The sensitivity increases by a factor of 17 in air $(1 g$ versus $2 g)$ and by over 400 in water ( $1 l$ versus $2 l)$. Limit of detection values are reduced by factors of 44 and 108 , respectively. Again we see that the advantages of SAB are more pronounced in the liquid environment.
TABLE IV

SENSITIVITY AND LOD FOR DETECTION OF TOLUENE IN AIR OR WATER

\begin{tabular}{|c|c|c|c|c|c|c|}
\hline Case & $1 g$ & $2 g$ & $3 g$ & $1 l$ & $2 l$ & $3 l$ \\
\hline Mode & WAB & SAB & WAB & WAB & SAB & WAB \\
\hline$S(\mathrm{~Hz} / \mathrm{ppm})$ & 0.008 & 0.138 & 0.137 & 0.095 & 38.8 & 5.70 \\
\hline LOD $(\mathrm{ppm})$ & 39 & 0.88 & 8.8 & 40 & 0.37 & 6.3 \\
\hline
\end{tabular}

- When frequency effects are discounted, the results indicate that sensitivity remains unchanged in air $(2 g$ versus $3 g)$ and increases tenfold in water $(2 l$ versus $3 l$ ) when using the $\mathrm{SAB}$ mode instead of WAB. The LOD advantages of SAB over WAB are demonstrated by the reduction factors of 10 and 17 in gas and liquid, respectively. All of these results are qualitatively consistent with the normalized results discussed previously (Table II) and the earlier explanations therefore apply here as well. We again conclude that the performance of the microsensor in SAB mode is superior to that in WAB mode, even for identical operational frequencies. The advantage is even more pronounced in liquid.

\section{SUMmary AND CONCLUSIONS}

A theoretical examination of the relative merits of strong-axis and weak-axis bending modes (SAB and WAB) in microcantilever sensors has been presented. Both gaseous and liquid environments have been considered. The main conclusion that may be drawn from this preliminary research is that the SAB mode has advantages in performance over the WAB mode because of the following.

a) The structure is stiffer in SAB mode (due to the larger cross-sectional moment of inertia $I$ ), resulting in a higher resonant frequency.

b) The SAB mode involves smaller viscous losses in the fluid, i.e., a higher quality factor and consequently a lower value of oscillator noise.

c) The SAB mode results in a smaller amount of fluid mass being dragged along with the microcantilever (due to the beam's more streamlined orientation as it moves through the fluid).

These advantages are reflected in improved values of sensitivity and detection limit and, as expected, these advantages are significantly more prominent in liquids than in gases, provided that the SAB mode can be implemented in an efficient manner.

For the practical implementation of the SAB mode, two possibilities can be considered.

1) The microcantilever may be fabricated with a thickness larger than the width, so that SAB operation (vertical vibration) may be activated by a vertical excitation. In this case, the methods of excitation would be similar to those employed in existing microcantilever technology.

2) A "conventional" microcantilever may be fabricated, i.e., with thickness less than the width. The lateral (horizontal) bending mode (SAB) may then be excited by either a) applying forces in the horizontal plane or $b$ ) applying vertical forces by standard means, but inducing a lateral component of vibration through the use of an eccentrically placed mass. 
While the latter approach may result in coupled vibrations involving not only the lateral (SAB) mode but possibly higher order vertical (WAB) and torsional vibrations, the practicality of this method has recently been demonstrated in the literature [25].

\section{REFERENCES}

[1] N. V. Lavrik, M. J. Sepaniak, and P. G. Datskos, "Cantilever transducers as a platform for chemical and biological sensors," Rev. Sci. Instrum., vol. 75, pp. 2229-2253, 2004

[2] M. Sepaniak, P. Datskos, N. Lavrik, and C. Tipple, "Microcantilever transducers: A new approach in sensor technology," Anal. Chem., pp. 568A-575A, Nov. 2002.

[3] O. Brand and H. Baltes, "Micromachined resonant sensors and overview," Sensors Update, vol. 4, pp. 3-51, 1998.

[4] B. Ilic, D. Czaplewski, H. G. Craighead, P. Neuzil, C. Campagnolo, and C. Batt, "Mechanical resonant immunospecific biological detector," Appl. Phys. Lett., vol. 77, no. 3, pp. 450-452, 2000.

[5] M. Godin, V. Tabard-Cossa, P. Grütter, and P. Williams, "Quantitative surface stress measurements using a microcantilever," Appl. Phys. Lett., vol. 79 , no. 4 , pp. $551-553,2001$

[6] C. Grogan, R. Raiteri, G. M. O'Connor, T. J. Glynn, V. Cunningham, M. Kane, M. Charlton, and D. Leech, "Characterisation of an antibody coated microcantilever as a potential immuno-based biosensor," Biosens. Bioelectron., vol. 17, pp. 201-207, 2002.

[7] K. B. Brown, W. Allegretto, F. E. Vermeulen, and A. M. Robinson, "Simple resonating microstructures for gas pressure measurement," $J$. Micromech. Microeng., vol. 12, pp. 204-210, 2002.

[8] B. Ilic, D. Czaplewski, M. Zalalutdinov, H. G. Craighead, P. Neuzil, C. Campagnolo, and C. Batt, "Single cell detection with micromechanical oscillators," J. Vacuum Sci. Technol. B, vol. 19, no. 6, pp. 2825-2828, 2001.

[9] M. Maute, S. Raibe, F. E. Prins, D. P. Kern, H. Ulmer, U. Weimar, and W. Göpel, "Detection of volatile organic compounds (VOCs) with polymer-coated cantilevers," Sens. Actuators B, vol. 58, pp. 505-511, 1999.

[10] T. A. Betts, C. A. Tipple, M. J. Sepaniak, and P. G. Datskos, "Selectivity of chemical sensors based on micro-cantilevers coated with thin polymer films," Anal. Chim. Acta, vol. 422, pp. 89-99, 2000.

[11] A. Boisen, J. Thaysen, H. Jensenius, and O. Hansen, "Environmental sensors based on micromachined cantilevers with integrated read-out," Ultramicroscopy, vol. 82, pp. 11-16, 2000.

[12] F. R. Blom, S. Bouwstra, M. Elwenspoek, and J. H. J. Fluitman, "Dependance of the quality factor of micromachined silicon beam resonators on pressure and geometry," J. Vacuum Sci. Technol. B, vol. 10, no. 1, pp. 19-26, 1992 .

[13] K. Y. Yasumura, T. D. Stowe, E. M. Chow, T. Pfafman, T. W. Kenny, B. C. Stipe, and D. Rugar, "Quality factors in micron- and submicron-thick cantilevers," J. Microelectromech. Syst., vol. 9, pp. 117-125, 2000.

[14] J. W. M. Chon, P. Mulvaney, and J. E. Sader, "Experimental validation of theoretical models for the frequency response of atomic force microscope cantilever beams immersed in fluids," J. Appl. Phys., vol. 87, pp. 3978-3988, 2000.

[15] J. M. Gere and S. P. Timoshenko, Mechanics of Materials, 4th ed. Boston, MA: PWS, 1997.

[16] H. Hosaka, K. Itao, and S. Kuroda, "Damping characteristics of beamshaped micro-oscillators," Sens. Actuators A, vol. 49, pp. 87-95, 1995.

[17] J. E. Sader, "Frequency response of cantilever beams immersed in viscous fluids with applications to the atomic force microscope," J. Appl. Phys., vol. 84, pp. 64-76, 1998

[18] R. D. Blevins, Formulas for Natural Frequency and Mode Shape. New York: Van Nostrand Reinhold, 1979.

[19] R. Yahiaoui and A. Bosseboeuf, "Improved modeling of the dynamical behaviour of cantilever microbeams," in MME 2001, Cork, Ireland, Sep. 16-18, 2001, pp. 281-284.

[20] J. W. Grate, A. Snow, D. S. Ballantine, Jr, H. Wohltjen, M. H. Abraham, R. A. McGill, and P. Sasson, "Determination of partition coefficients from surface acoustic wave vapor sensor responses and correlation with gas-liquid partition coefficients," Anal. Chem., vol. 60, pp. 869-875, 1988.

[21] I. Dufour, F. Lochon, S. M. Heinrich, F. Josse, and D. Rebière, "Effect of coating viscoelasticity on quality factor and limit of detection of microcantilever chemical sensors," IEEE Sensors J., vol. 7, no. 2, pp. 230-236, Feb. 2007.
[22] D. Lange, C. Hagleitner, A. Hierlemann, O. Brand, and H. Baltes, "Complementary metal oxide semiconductor cantilever arrays on a single chip: Mass-sensitive detection of volatile organic compounds," Anal. Chem., vol. 74, pp. 3084-3095, 2002.

[23] R. A. McGill, "Choosing polymer coatings for gas and liquid chemical microsensors," in Proc. SPIE Soc. Plastics Eng. Annu. Tech. Conf., May 5-10, 1996, pp. 2080-2084.

[24] A. Hierlemann, E. Zellers, and A. Ricco, "Use of linear solvation energy relationships for modeling responses from polymer-coated acoustic-wave vapor sensors," Anal. Chem., vol. 73, pp. 3459-3466, 2001.

[25] L. B. Sharos, A. Raman, S. Crittenden, and R. Reifenberger, "Enhanced mass sensing using torsional and lateral resonances in microcantilevers," Appl. Phys. Lett., vol. 84, pp. 4638-4640, 2004.

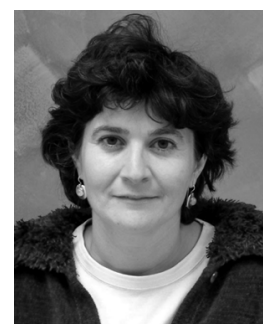

Isabelle Dufour received the Agrégation de Sciences Physiques, Ph.D., and Habilitation à Diriger des Recherches degrees from the University of Paris Sud Orsay, France, in 1989, 1993, and 2000, respectively.

Her work was on the use of magnetic sensors for nondestructive testing and the modeling of microactuators. She is presently a Researcher with the Centre National de la Recherche Scientifique at the IXL Microelectronic Laboratory. Her research is now focused on chemical microsensors using moving structures.

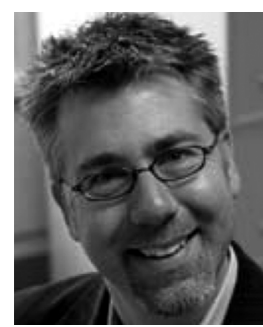

Stephen M. Heinrich received the B.S. degree (summa cum laude) from Pennsylvania State University, State College, in 1980 and the M.S. and $\mathrm{Ph} . \mathrm{D}$. degrees from the University of Illinois at Urbana-Champaign in 1982 and 1985, respectively, all in civil engineering.

He then took an academic position at Marquette University, Milwaukee, WI, where he is currently Professor of civil engineering. Throughout the $1990 \mathrm{~s}$, his research focused on structural mechanics applications in microelectronics packaging, including the development of analytical models for predicting solder joint geometries and the thermomechanical deformations of soldered microelectronic structures. In more recent years, he has investigated new analytical models for predicting/enhancing the performance of micro/nanocantilever sensors. His work has resulted in approximately 90 publications/presentations.

Dr. Heinrich has received three Best Paper Awards from IEEE and ASME. In 2000, he received Marquette University's highest teaching honor, the Reverend John P. Raynor Faculty Award for Teaching Excellence. In 2006, Dr. Heinrich was awarded a Professeur Invité position and a Fillbright-Aquitaine Research Scholor Award at Laboratoire IXL, Université Bordeaux 1, France. He has been a member of the ASCE Elasticity Committee and Associate Editor of the IEEE TRANSACTIONS ON ADVANCED PACKAGING and ASME Journal of Electronic Packaging.

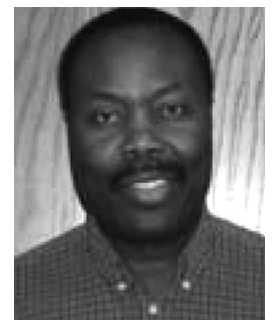

Fabien Josse (SM'93) received the License de Mathematics et Physique from the Université du Benin in 1976 and the M.S. and Ph.D. degrees in electrical engineering from the University of Maine at Orono in 1979 and 1982, respectively.

He has been with Marquette University, Milwaukee, WI, since 1982 . He is currently a Professor in the Department of Electrical and Computer Engineering and the Department of Biomedical Engineering, as well as Director of Graduate Studies. $\mathrm{He}$ is an Adjunct Professor in the Department of Electrical and Computer Engineering, Laboratory for Surface Science and Technology, University of Maine. He has been a Visiting Professor at the University of Heidelberg, Germany, since 1990 and a Visiting Professor with Laboratoire IXL, University of Bordeaux, France, and the Physical Electronics Laboratory, Swiss Federal Institute of Technology, Zurich. His current research interests include solid-state and acoustic wave device sensors (liquid-phase chemical and biochemical sensors), microelectromechanical systems devices (microcantilevers for sensor applications), investigation of novel chemical and biochemical sensor platforms, and smart sensor systems.

Dr. Josse is a member of Eta Kappa Nu and Sigma Xi. He is an Associate Editor of the IEEE SENSORS JOURNAL. 